\title{
A Rendezvous Node Selection Algorithm for Mobile Sink Path Planning in Wireless Sensor Networks
}

\author{
Xiaoming $\mathrm{Wu}^{1,2,3+}$, Yinglong Wang ${ }^{1,2,3}$ and Yifan $\mathrm{Hu}^{2,3}$ \\ ${ }^{1}$ College of Information Science and Engineering, Shandong University of Science and Technology, \\ Qingdao, China \\ ${ }^{2}$ Shandong Computer Science Center (National Supercomputer Center in Jinan), Jinan, China \\ ${ }^{3}$ Shandong Provincial Key Laboratory of Computer Network, Jinan, China
}

\begin{abstract}
Recent research shows that significant energy hole problem can be solved in wireless sensor network (WSN) by using mobile sink capable of carrying data. In order to plan an optimal moving tour for mobile sink without long distance travel, we develop the rendezvous node selection algorithm (RNSA) to find rendezvous nodes (RN) for the sink to visit. The RNSA can select the set of RNs to act as store points for the mobile sink, and several factors would be considered to find suitable RNs from sensor nodes, the other sensor nodes would forward data via multi hopping to the nearest $\mathrm{RN}$, such that the energy consumption of sensor nodes is minimized and uniform to avoid the problem of energy holes while ensuring sensed data are collected on time. Our scheme is then validated through extensive simulations.
\end{abstract}

Keywords: path plan, wireless sensor network, mobile sink, rendezvous node, energy hole.

\section{Introduction and Related Work}

Wireless sensor network (WSN) technology is the result of recent developments in the field of microelectronic mechanical systems (MEMS) and wireless communication technology that has resulted in the construction of small and smart sensors. Sensors are deployed in a wireless network and are used in civilian applications such as smart city, industrial control and environmental monitoring [1]. WSN in these applications often produce high-bandwidth sensor data that need to be collected under stringent delay constraints.

However, in previous work, mobility and communication capabilities are often underutilized, resulting in suboptimal solutions incurring unnecessarily energy hole and large latency [2]. In this paper, we would focus on the problem of finding an optimal moving path of the sink, to achieve the smallest data delivery latency in the case of reducing energy consumption at each sensor.

Several studies have demonstrated the benefits of using a mobile sink to reduce the energy consumption of nodes and to avoid problem of energy holes in WSN [3]. The mobile sink moves in the sensed area to collect the data. It must return to the base station before a deadline because of the limited running time of mobile sink. However, these benefits are dependent on the path taken by the mobile sink, as all sensed data should be collected within a given time constraint. A scheme proposed to address this challenge is to form a moving tour for mobile sink [4]. During travel, the mobile sink passes temporarily through some places called rendezvous nodes (RN) to collect data without traveling long distances [5]. The RN would buffer data originated from source nodes and transfer to the sink when it arrive. If Sensor nodes are not RNs then forward its sensed data via multi hopping to the nearest RN. Our goal is to decide the location of the

\footnotetext{
+ Corresponding author. Tel.: + 8615963135456.

E-mail address:wuxm@sdas.org.
} 
rendezvous nodes so that the energy consumption of the WSN is reduced and the mobile sink can return to the base station in time.

For the static sink WSN, a TSP-based algorithm [6] called label-covering algorithm (LCA) considers a WSN as a complete graph. For each edge, it calculates a cost and associates a label set. The cost of an edge is the Euclidean distance between sensor nodes, whereas the label set contains sensor nodes whose transmission range intersects with the given edge. The label-covering algorithm selects the minimum number of edges where their associated label set covers all sensor nodes. But this scheme is only applied in the static sink network, which would be compared to our mobile sink network scheme in our simulation.

In [7], to have better energy saving of WSN, the authors plan rendezvous points for a mobile sink to travel by mapping sensors in a plane to a point on a Halin graph. They focus on balancing energy depletion to extend network lifetime with the use of mobile sinks and a proper travel plan. The scheme provides a protocol to plan rendezvous points for the travelling of mobile elements. In the selecting procedure, they consider the deadline and the shortest path for mobile sink to travel those points. Using the Halin graph helps solve rendezvous planning in polynomial time.

A heuristic WRP (Weighted rendezvous planning) algorithm is proposed in [8]. Here mobile-sink node only visits rendezvous nodes (RNs). The basic problem is computing a tour that visits all these RNs. If Sensor nodes are not RNs then forward its sensed data via multi hopping to the nearest RN. Each sensor node is assigned a weight depending on the hop distance from the tour and the number of data packets. Simulation results demonstrate that WRP enables a mobile sink to retrieve all sensed data within a given deadline while conserving the energy expenditure of sensor nodes.

However, WRP [8] is not suitable for the large scale sensor networks, as when the nodes number is too large, the complexity of RN selection optimization problem would be too difficult. Besides, in the problem of selecting RN, the WRP only considered factors of distance between the alternative RN and RNs set, and the stored date number of alternative RN, but not the remaining energy and its neighbors, which is not comprehensive.

To address the rendezvous node (RN) selecting problem, we develop a Rendezvous Node Selection Algorithm (RNSA) to optimize the mobile sink path planning scheme in the WSN. Our proposed scheme differs from the above works. In this algorithm, 4 factors would be considered in the RN selecting process: the remaining energy of the alternative RN, its neighbors' number, stored delivery packet number, and its distance to other RNs. The main contributions of this paper are as follows:

1) We develop the rendezvous node selection algorithm (RNSA) to select a set of RNs and plan the sink moving path, in which the RN can act as a store point for the mobile sink to visit. We also define the several factors to find optimal RNs, in order to balance energy consumption and avoid the energy holes problem.

2) We analyze and compare our protocol and WRP, LCA in terms of energy consumption and packet delivery. Simulation results show the superiority of the protocol.

The rest of this paper is organized as follows. Section 2 describes our mobile sink network model and the proposed algorithm in details. Section 3 evaluates the performance of the RNSA by comparing it with other routing protocols. Finally, the conclusion is presented in Section 4.

\section{Description of Network Model}

\subsection{System Models and Assumptions}

Our network model is a connected graph $G(V, E)$ [9], where $V$ is a finite set of sensor nodes and $E$ is the set of edges representing connection between these nodes. In the network model as shown in Figure 1, $v_{s}^{i}$ $(0<i<N)$ is the source node, $v_{R N}^{j}(0<j<N)$ is the rendezvous node, and $v_{\operatorname{sink}}$ is the mobile sink. Suppose there exist $m(0<m<N-1)$ source nodes and one mobile sink, these source nodes $\left(v_{s}^{1}, v_{s}^{2}, v_{s}^{3} \ldots v_{s}^{6}\right)$ would route the packets through multi-hops relay node path to the $\mathrm{RN} v_{R N}^{j}$, then $v_{R N}^{j}$ stores the packets, and transmits to the mobile sink when the sink moves close to its communication range, as illustrated in Figure 1. Sensor nodes that are not RNs forward their sensed data via multi hopping to the nearest RN. The fundamental problem then becomes identifying the optimal RNs that the sink would visit all these RNs 
within a given delay bound, and planning moving path for the sink.

Our network model is based on the following assumptions: 1) The area is covered by a large number of homogeneous sensor nodes. Sensor nodes are stationary, but the sink moves constantly with a relatively fixed speed. 2) Nodes and sink are aware of their physical locations. 3) Data is sensed and delivered from each source node to the nearest RN every $T$ time period.

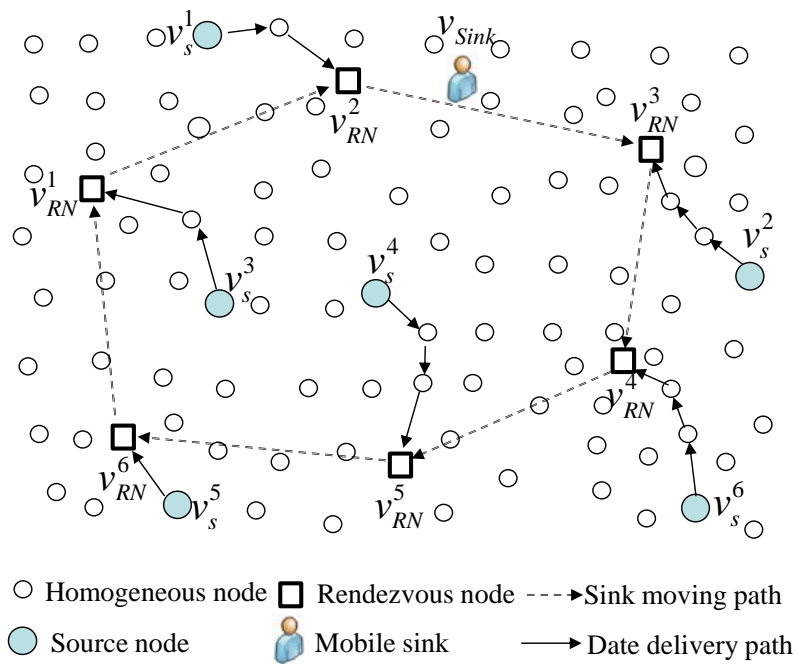

Fig. 1: The example of sink moving path in the WSN.

\subsection{Rendezvous Point Selection Algorithm}

The following RN selecting algorithm can calculate and decide the optimal and suitable RN from the sensor nodes, in order to update and maintain the dynamic network routing from source node to the sink.

1) Global selecting RN (rendezvous node). We would set the density of $\mathrm{RN} \operatorname{Dens}\left(v_{R N}\right)=40$, which means there would be one $\mathrm{RN}$ among 40 sensor nodes, then the number of $\mathrm{RN}$ in the network is $\operatorname{NumV}\left(v_{R N}\right)=\operatorname{Num} V(V) / \operatorname{Dens}\left(v_{R N}\right)$. The factors affecting the choice of RNs $v_{R N}^{i}$ contain 4 factors after normalization:

a) Leng $\left(H, v_{R N}^{i}\right)$, distance rate of $v_{R N}^{i}$ to other RPs, is the ratio of the distance between $v_{R N}^{i}$ and the other selected RN set $H\left(\operatorname{Sink}, v_{R N}^{j} \cdots\right)(0<j<M a x, j \neq i)$ to the maximum length of sink moving path $D$ :

$$
\operatorname{Leng}\left(H, v_{R N}^{i}\right)=\operatorname{Leng}\left(H, v_{R N}^{i}\right) / D
$$

b) $\operatorname{Num} P\left(v_{R N}^{i}\right)$, the packet delivery rate, is the ratio of $v_{R N}^{i}$ 's packet storage number to the max packet storage number:

$$
\operatorname{NumP}\left(v_{R N}^{i}\right)=\operatorname{StoreP}\left(v_{R N}^{i}\right) / \operatorname{Max} \operatorname{Store} P\left(v_{R N}^{i}\right)
$$

c) Ene $\left(v_{R N}^{i}\right)$, the remaining energy rate, is the ratio of $v_{R N}^{i}$ 's remaining energy to its initial energy:

$$
\operatorname{Ene}\left(v_{R N}^{i}\right)=\operatorname{Re} \text { maining Ene }\left(v_{R N}^{i}\right) / \operatorname{Initial} \operatorname{Ene}\left(v_{R N}^{i}\right)
$$

d) $\operatorname{Neigh}\left(v_{R N}^{i}\right)$, the neighbor node rate, is the ratio of $v_{R N}^{i}$ 's neighbor nodes number to the whole network nodes number:

$$
\operatorname{Neigh}\left(v_{R N}^{i}\right)=\operatorname{NeighNum}\left(v_{R N}^{i}\right) / \operatorname{NodeNum}\left(v_{R N}^{i}\right)
$$

The fitness function of node $f i t\left(v_{R N}^{i}\right)$ is:

$$
\operatorname{fit}\left(v_{R N}^{i}\right)=\omega_{1} \operatorname{Leng}\left(H, v_{R N}^{i}\right)+\omega_{2} \operatorname{NumP}\left(v_{R N}^{i}\right)+\omega_{3} \operatorname{Ene}\left(v_{R N}^{i}\right)+\omega_{4} \operatorname{Neigh}\left(v_{R N}^{i}\right) \text { s.t. } \sum^{N} \operatorname{Leng}\left(H, v_{R N}^{i}\right)<D, \quad \omega_{1}+\omega_{2}+\omega_{3}+\omega_{4}=1
$$

where $\omega_{1}, \omega_{2}, \omega_{3}, \omega_{4}$ are the weight of distance rate, packet dellivery rate, remaining energy rate, and neighbor node rate in the fitness function. We set $\omega_{1}=0.2, \omega_{2}=0.1, \omega_{3}=0.4, \omega_{4}=0.3$, which can enhance the importance of remaining energy and neighbor node number on the RN selection. D is the maximum length constraint of sink moving path for the $\mathrm{RN} v_{R N}^{i}$. 
After all the RNs are selected, our proposed algorithm would call the TSP solver to calculate a Sink moving tour that visits all the RNs, so that the sink can plan the shortest tour length to traverse all the RNs. In our algorithm and simulation, we used a local-search-based heuristic TSP solver as mentioned in [10].

2) Alternative RN selection: If the remaining energy of $v_{R N}^{i}$ is less than min value or it is failed, one of alternative RNs in the one hop neighbor of the previous RN would be selected, the process is the same as step 1). If suitable $\mathrm{RN}$ is selected, the moving path of the mobile sink would not be changed; otherwise, the alternative $\mathrm{RN}$ would be selected in the local range around the previous $\mathrm{RN}$, and the moving path of the sink would also be changed, then the new shortest moving path of sink is calculated and considered as its new moving plan.

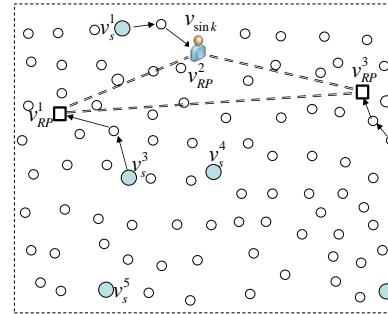

(a)

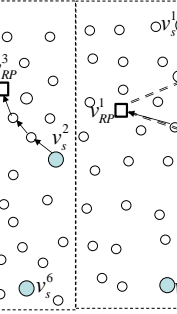

(b)
Source node

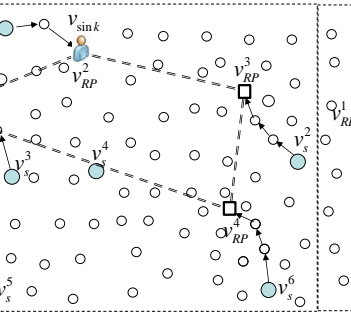

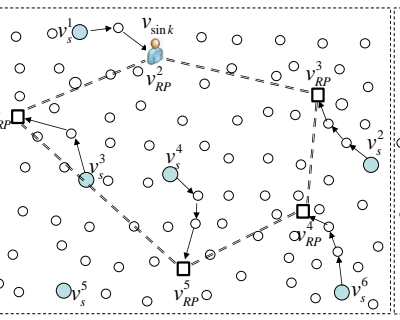

(c)

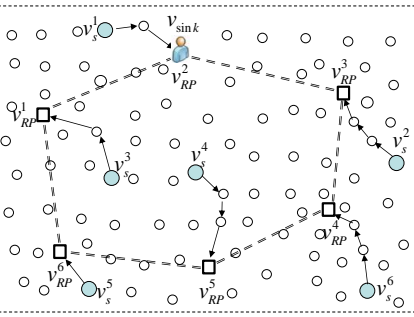

(d)

Fig. 2: The proposed rendezvous node selection algorithm step.

An example shown in Figure 2 shows the process of our RNSA selecting the suitable RNs in the network. In Fig. 2(a), the sink starts to find the third RN with optimal fitness value in the equation (5), and construct the path of $\left\{v_{R N}^{2}, v_{R N}^{3}, v_{R N}^{1}\right\}$. In Fig. 2(b), Our RNSA selects node $v_{R N}^{4}$ with highest fitness value, and the TSP solver would return the path of $\left\{v_{R N}^{2}, v_{R N}^{3}, v_{R N}^{4}, v_{R N}^{1}\right\}$, which is less than the distance $D$. Therefore, node $v_{R N}^{4}$ is added to the tour. The process continues, yielding a final tour of $\left\{v_{R N}^{2}, v_{R N}^{3}, v_{R N}^{4}, v_{R N}^{5}, v_{R N}^{6}\right.$, $\left.v_{R N}^{1}\right\}$ with a tour length $D^{\prime}$, which is less than the required tour length, else if the tour length $D^{\prime}>\mathrm{D}$, the node would be given up, and we would reselect another suitable RN. As shown in Fig. 2(d), the final tour computed by our RNSA always includes sensor nodes as RNs that have more data packets to forward, more neighbor nodes, more remaining energy, and more suitable distance to each other in the network. This ensures the energy consumption balance and can solve the energy-hole problem.

In the network energy model [11], the equation of the node energy model is as follow:

$$
\text { ene }(l, d)=\text { ene }_{t x}(l, d)+\text { ene }_{r x}(l)=\left(a_{11}+a_{2} d^{n}\right) l+a_{12} l
$$

where $d$ is the distance from the node to the next-hop node, ene $e_{t x}(l, d)$ and ene $e_{r x}(l)$ are the energy consumption of sending and receiving $l$ bits of data, $n$ is the channel attenuation index, $a_{11}, a_{2}$ and $a_{12}$ are energy consumption parameters of sending circuit, sending amplifier and receiving circuit. Using this model, the energy consumption of the data delivery can be calculated in the simulation.

\section{Experimental Evaluation of the Protocol}

\subsection{Model and Assumption}

We evaluated our RNSA (rendezvous node selection algorithm) with simulation-based experiments, the simulation was conducted in an environment developed based on Matlab 2008a. The experimental hardware environments are Intel ${ }^{\circledR} \mathrm{i} 7-4600 \mathrm{M}, 2.90 \mathrm{GHz}$ CPU and $4 G B$ memory, operating system is MS windows 7. The whole network is simulated in the area of $3500 \mathrm{~m} \times 3500 \mathrm{~m}$. The field is static and 30 300 sensor nodes are deployed uniformly in which $10 \%$ sensor nodes are source nodes. All sensor nodes have fully charged battery with $150 \mathrm{~J}$ of energy, the source node delivers packets at the rate of 20 data packets per round, with $10 \mathrm{~KB}$ of each packet size, and the simulation lasts for 300 rounds. In the energy model, Ene elec $=40 \mathrm{~nJ} / \mathrm{bit}$, $E n e^{a m p}=60 n \mathrm{~J} / \mathrm{bit}$. The mobile sink speed is $1 \mathrm{~m} / \mathrm{s}$. Our proposed RNSA was compared to WRP (weighted 
rendezvous planning) and LCA (label covering algorithm). The performance metrics used for the comparison are energy consumption, standard deviation of energy consumption and network lifetime.

In the simulation, we use standard deviation (SD) to measure the imbalance between the node's energy consumption, for example, a wider variation means some parts of the network are more likely to exhaust their energy soon. The metric SD is calculated as follows:

$$
S D=\sqrt{\frac{\sum_{\operatorname{Num}(V)}^{i=1}\left(\operatorname{ene}\left(v_{i}\right)-\mu\right)}{\operatorname{Num}(V)}}
$$

where ene $\left(v_{i}\right)$ is the energy consumption of node $v_{i}, V$ is the set of sensor nodes, $N u m(V)$ is the number of sensor nodes, and $\mu$ is the average energy consumption of sensor nodes.

\subsection{Evaluation of the Experimental Results}

Figure 3 shows the network energy consumption ratio for the 3 protocols. The RNSA achieves 10\% reduction in network energy consumption, as compared with WRP. The reason is that the RNSA can select the RNs with better QoS parameters (e.g. more residual energy, better distance to other RNs and more neighbor nodes) to form the sink moving path. Then it would construct a more reliable transmission environment to reduce the energy consumption of nodes.

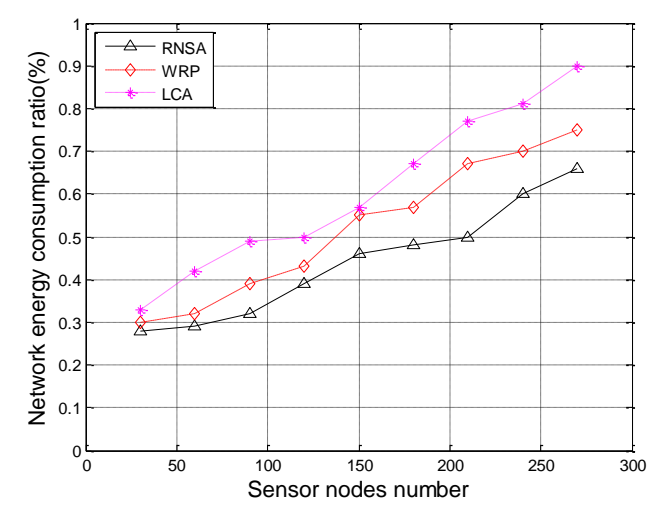

Fig. 3: Network energy consumption for RNSA, WRP and LCA.

The ability of the three protocols to uniformly distribute energy consumption is presented in Figure 4, which indicates the standard deviation of energy consumption ratio to the sensor nodes number. We can observe, our RNSA distributes energy more uniformly than the other two protocols (7\% more than WRP, and $15 \%$ better than LCA). Comparing with WRP, RNSA preferentially selects nodes with lower energy consumption rate and less hop count from the sink.

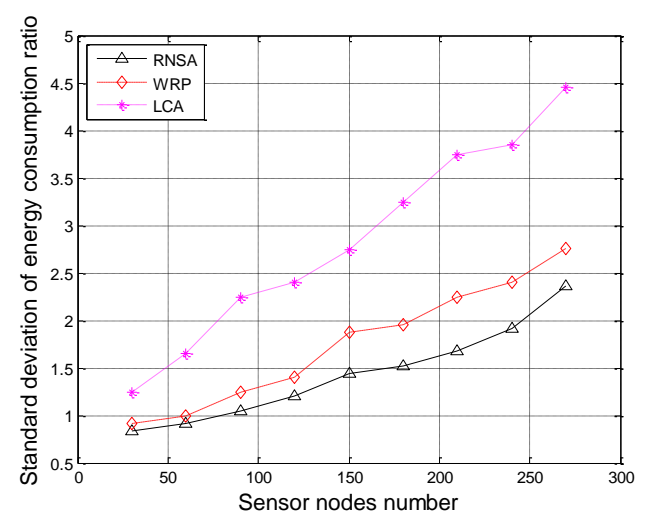

Fig. 4: Standard deviation of nodes energy consumption for RNSA, WRP and LCA.

We now evaluate the network lifetime performance of different algorithms. As shown in Figure 5, we observe that among the three protocols, RNSA serves a reduction of $5 \%$ and $14 \%$ on network lifetime 
compared to WRP and LCA, that is because when the RN is exhausted, another optimal RN would be selected in our algorithm, the network energy dispersion is balanced and it would take longer time for a node to die.

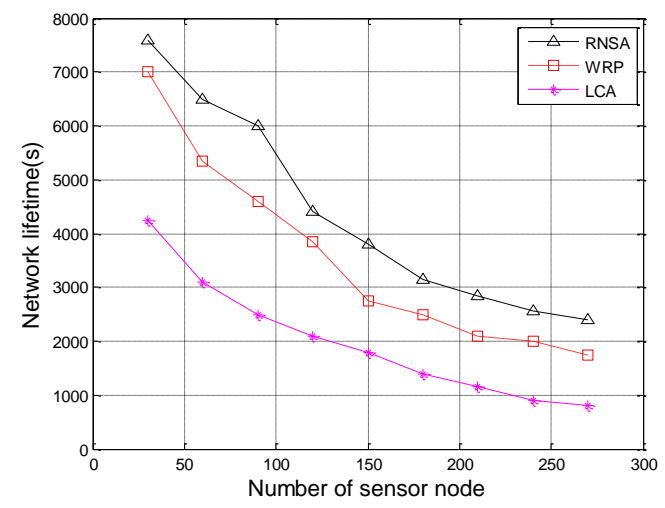

Fig. 5: Network lifetime for RNSA, WRP and LCA.

\section{Conclusion}

This study presents a novel rendezvous node selection algorithm for the mobile sink to visit the optimal RNs in the WSN, in order to plan an optimal moving tour for mobile sink without long distance travel. The proposed RNSA would find suitable RNs with optimal factors, then the other sensor nodes would forward data via multi hopping to the nearest RN, such that the energy consumption of sensor nodes is minimized and uniform to avoid the problem of energy holes while ensuring sensed data are collected on time. More importantly, this paper demonstrated the applicability and the potential of RNSA algorithm for solving RN selection optimization problems. The simulations show that, our RNSA reduces energy consumption by $7 \%$ and increases network lifetime by $6 \%$, as compared with existing algorithms. Our RNSA could efficiently solve the energy hole problem, balance the network traffic load, and maintain the network robustness against sink moving problem.

In the future we will focus on the multi-mobile sink network and extend our approach to heterogeneous WSN by taking advantage of the nodes with more computational power and storage capacity, and the most important optimization objective is maximizing the network lifetime.

\section{Acknowledgement}

This work was supported in part by the National Nature Science Foundation of China (No. 61401257, 61501282), Shandong Academy Young Scientists Fund Project (No. 2013QN037), Shandong Academy Basic Research Fund, and Shandong Academy of Sciences Pilot Project for Science and Technology.

\section{References}

[1] Kashif Saleem, Abdelouahid Derhab, Jalal Al-Muhtadi, Mehmet A. Orgun, Analyzing ant colony optimization based routing protocol against the hole problem for enhancing user's connectivity experience, Computers in Human Behavior, 2015, 51: 1340-1350.

[2] Y. S. Jiang, W. R. Shi, X. G. Wang and H. B. Li, “A distributed routing for wireless sensor networks with mobile sink based on the greedy embedding," Ad Hoc Networks, 2014, 20: 150-162.

[3] Y. F. Hu, Y. S. Ding, K. R. Hao and H. Han, "An endocrine cooperative particle swarm optimization algorithm for routing recovery problem of wireless sensor networks with multiple mobile sinks," Information Sciences, 2015 , 300: $100-113$.

[4] S. Mottaghi, M. R. Zahabi, Optimizing LEACH clustering algorithm with mobile sink and rendezvous nodes, AEU - International Journal of Electronics and Communications, 2015, 69 (2): 507-514.

[5] Ali Sharifkhani, Norman C. Beaulieu, A mobile-sink-based packet transmission scheduling algorithm for dense wireless sensor networks, IEEE Trans. Vehicular Technology, 2009, 58 (5): 2509-2518. 
[6] R. Sugihara and R. Gupta, Improving the data delivery latency in sensor networks with controlled mobility, Distributed Computing in Sensor Systems, 2008: 386-399.

[7] R. S. Chang, S. H. Wang, S. L. Tsai, W. P. Yang, Planning rendezvous using the Halin graph in wireless sensor networks, Wireless Sensor Systems, IET, 2012, 2 (3): 222-229.

[8] Salarian, H.; Kwan-Wu Chin; Naghdy, F., An Energy-Efficient Mobile-Sink Path Selection Strategy for Wireless Sensor Networks, IEEE Trans. Vehicular Technology, 2014, 63 (5): 2407-2419.

[9] G. L. Xing, T. Wang, Z. H. Xie, and W. J. Jia, Rendezvous Planning in Wireless Sensor Networks with Mobile Elements, IEEE Trans. Mobile Computing, 2008, 7 (12): 1430-1443.

[10] I. Cholissodin, To solving traveling salesman problem (TSP) for 10 till 100 city with local search, Dec. 2007.

[11] Y. F. Hu, Y. S. Ding, K. R. Hao, L. H. Ren and H. Han, An immune orthogonal learning particle swarm optimization algorithm for routing recovery of wireless sensor networks with mobile sink, International Journal of Systems Science, 2014, 45 (3): 337-350. 